\title{
JAK2 and Its Regulators beyond Myeloproliferative Neoplasms
}

\author{
Nahla A.M. Hamed* \\ Faculty of Medicine, Alexandria University, Egypt
}

Submission: January 06, 2018; Published: March 19, 2018

*Corresponding author: Nahla A.M. Hamed, Professor of Hematology, Faculty of Medicine, Alexandria University, Egypt, Email: drhamedn@hotmail.com

\begin{abstract}
Dysregulated JAK2 is at the apex of three classic oncogenic signaling cascades including STAT, PI3K/Akt/mTOR, and MAPK pathways. Their interrelationship and dependencies downstream of JAK2 in MPN cells is incompletely understood. Combined pathway inhibition in vitro might prove beneficial due to a synergistic impact on functionally redundant oncogenic axes, might allow lower doses of the different agents with better tolerability, and might avoid or delay the development of resistance.

Abbreviations: JAK: Janus Family of Kinases; STAT: Signal Transducer and Activator of Transcription Factors; MF: Myelofibrosis; MPN: Myeloproliferative Neoplasm; MDS: Myelodysplastic Syndrome; ET: Essential Thrombocythemia; BAT: Best Available Therapy; TNF: Tumor Necrosis Factor; IFN: Interferon; MAPK: Mitogen-Activated Protein Kinases; PI3K: Phosphatidylinositol-3'-Kinase; SOCS: Suppressor of Cytokine Signaling
\end{abstract}

\section{Introduction}

\section{JAK-STAT signaling pathway}

The JAK-STAT pathway constitutes a signal transduction system through which a large spectrum of extracellular cytokines and nearly as many cognate transmembrane receptors converge towards an intracellular code employing four JAK kinases (JAK1, JAK2, JAK3, and TYK2) and seven STAT factors (STAT-1, $-2,-3,-4,-5 a,-5 b$ and -6 ) [1]. The activated STATs dimerize and translocate into the cell nucleus to influence DNA transcription, and regulating gene expression. The JAK-STAT pathways activate or suppress the transcription of a wide array of genes that affect cell growth and apoptosis such as SOCS, Nmi, Bcl-XL, p21, MYC, and NOS2 [2]. Each member of the JAK family has a primary role in mediating a signaling process with some overlap between them. JAK2 is important for hematopoietic growth factors signaling such as Epo, GM-CSF, thrombopoietin, IL-3, IL-5, growth hormone and prolactin-mediated signaling [3].

\section{JAK2 and its regulators beyond MPNs}

Hyperactive JAK signaling is found in MPNs such as polycythemia vera, ET, and MF, $7.8 \%$ to $13 \%$ of chronic myelomonocytic leukemia and $1 \%$ to $4.2 \%$ of MDS. JAK2 (V617F)-positive AML is mostly observed in patients with a previous MPN, but cases of V617F in de novo AML have been described. A gain-of-function mutation in the kinase domain, T875N, was reported in acute megakaryoblastic leukemia. JAK2 mutations are relatively rare in lymphoid malignancies except for ALL, most commonly in Down syndrome-associated ALL. JAK1, JAK2, and JAK3 mutations and translocations are observed in high-risk BCR-ABL B-cell ALL. Amplification of the JAK2 locus has been detected in Hodgkin lymphoma and mediastinal B-cell lymphoma. JAK2 mutations and translocations are very rare in solid cancers [4].

\section{JAK inhibitors}

Type I JAK inhibitors precisely target the well-conserved ATP-binding pocket of JAK1 and JAK2 in both active and inactive conformations. Ruxolitinib (and other JAK inhibitors under clinical evaluation) falls within the type I family [1] which has insufficient efficacy and limited JAK inhibition potential [4]. Their lack of selectivity implies that the tolerable doses lie within a narrow therapeutic window because of the collateral inhibition of other JAK members. Specific inhibitors for one of the four closely related JAK kinases could partially tackle this drawback because of the marked similarity in the active site within the family. Inhibitors that have a type II binding-mode specifically engage and stabilize inactive kinases by exploiting an additional, less conserved allosteric site directly adjacent to 
the ATP binding pocket, providing another handle for tuning selectivity. Alternatively, a covalent inhibitor exploiting a nonconserved cysteine residue in the active site of JAK3 has been shown to have strong target specificity [1].

\section{The JAK1 and JAK2 inhibitor, ruxolitinib}

Myelofibrosis: Ruxolitinib is the only JAK inhibitor approved for treatment of patients with MF. The drug mainly inhibits dysregulated JAK-STAT signaling, present in all MF patients irrespective of their JAK2 mutational status. It is not selective for the mutated JAK2, which explains its efficacy in both JAK2positive and JAK2-negative MF [5]. Of note, clinical responses in MF patients were linked to suppression of increased serum levels of proinflammatory cytokines such as IL-1, IL-6, TNF- $\alpha$ and IFN- $\gamma$ [6]. Ruxolitinib has become the standard of care for intermediate or high-risk patients with MF; it provides rapid and sustained reduction of splenomegaly and improvement in symptoms and quality of life, possibly resulting in prolongation of survival [5].

Ruxolitinib treatment failure in MF manifests as primary refractoriness or secondary resistance. Primary refractoriness can be defined as no or only minimal clinical response $(<35 \%$ reduction of spleen volume compared to baseline), whereas secondary resistance is indicated by the loss of a previously confirmed clinical response (such as splenic relapse) or progression to leukemia [1]. Long-term follow-up showed that about half the patients enrolled in the COMFORT-I and COMFORT-II studies discontinued treatment by 3 years, largely because of loss of response and/or disease progression [5]. Primary refractoriness is rare $(<10 \%)$ [1]. The mechanisms of resistance and/or loss of response to ruxolitinib have not yet been deciphered [7]. It could be expected that resistant patients would have acquired mutations at the drug-binding site of the target kinase [1]. Acquisition of mutations in the predicted ruxolitinib-binding region conferred resistance to JAK inhibitors, have not been documented in patients [7]. A significant correlation between resistance and the absence of mutations in JAK2 (V617F), MPL, TET2, and SRSF2 at diagnosis has been identified [1].

The phenomenon of persistence to a JAK2 inhibitor (i.e., the fact that JAK2 (V617F) mutated cells survive despite chronic JAK inhibition) was ascribed to heterodimerization between activated JAK2 and other members of the JAK family and was shown to occur in patients treated with JAK2 inhibitors; however, the clinical relevance of such observations remains unsettled [7].

\section{Limitations of Type I JAK Inhibition in MF}

There are several limitations to the use of JAK2 inhibitors in MF patients. First, the safety and clinical efficacy of ruxolitinib are unclear in patients present with platelet counts between $50 \times 10^{9} / \mathrm{l}$ to $100 \times 10^{9} / \mathrm{l}$ or even $<50 \times 10^{9} / \mathrm{l}$, and in patients present with transfusion-dependent anemia, since ruxolitinib have platelet lowering properties and can lead to anemia in some patients. Second, it does not cure the disease and requires continuing therapy to maintain response [3]. Besides, there is no biological evidence of a possible favorable effect of ruxolitinib on the survival of MF patients, such as the achievement of a complete or a partial remission, cytogenetic or molecular response, or reversal of the bone marrow fibrosis [5]. Lastly, the response may be short lived in some patients and there are reports of adverse events occurring at the time of withdrawal [3].

\section{Polycythemia Vera and Essential Thrombocythemia}

Ruxolitinib is also approved for the second-line treatment of polycythemia vera and is being developed for ET [8]. Patients with ET who are refractory to or intolerant of hydroxyurea can achieve clinically meaningful and durable reductions in platelet and WBC counts and improvements in ET-related symptoms with ruxolitinib treatment [9]. However, ruxolitinib did not improve treatment efficacy for most clinically relevant events compared with BAT and was more frequently associated with anemia and infections than was BAT [10].

\section{Other Indications}

Ruxolitinib is approved for MDS and is effective in reducing splenomegaly. The medication is off-label for hypereosinophilic syndrome, a report demonstrates successful treatment of HES patients, who are intolerant to other treatments, with ruxolitinib [11]. The efficacy of ruxolitinib in GVHD was reported in a murine model, and its potent activity was demonstrated in 6 patients with corticosteroid-refractory GVHD. Findings demonstrate that targeting JAK1/2 signaling in alloreactive $\mathrm{T}$ cells is a powerful approach to GVHD inhibition. The key event is inhibition of STAT3 phosphorylation by ruxolitinib during an allogeneic immune response. In addition to, the drugspecific effects on Treg development. Unlike, most conventional immunosuppressive agents which target T-cell function, ruxolitinib impair differentiation, maturation, and cytokine production of dendritic cells, which may further increase its efficacy in GVHD. Suppression of proinflammatory cytokines such as IL-1b, IL-6, or IFN- $\gamma$ which are considered hallmarks of GVHD could potentially reduce disease severity [6].

\section{Alternative Options to Target Myeloproliferative Neoplasms}

Other JAK inhibitors with the potential for significantly less myelosuppression or even improvement of anemia continue to be tested [8]. Type II JAK inhibition such as BBT594 can overcome persistence to ruxolitinib in JAK2 (V617F)- and MPL W515L-positive cells. In contrast with type I JAK inhibition, inactive JAK2 is stabilized and activation loop phosphorylation is decreased [4].

Several alternative options to target MPNs are being explored. HSP90 inhibitors such as PU-H71 and AUY922 efficiently impair JAK-mediated signaling and overcome resistance due to secondary mutations. Histone deacetylase inhibitors, including panobinostat, reduce JAK2 expression most probably due to 
effects on JAK2 mRNA expression, and increased proteasomal JAK2 degradation. In vitro studies suggest synergistic effects of PI3K/mTOR inhibitors or of the MEK1/2 inhibitor with JAK2 inhibitors. Combined pathway inhibition therapy might prove beneficial due to a synergistic impact on functionally redundant oncogenic axes, might allow for lower doses of the different agents with better tolerability, and might avoid or delay the development of resistance [4].

\section{Conclusion}

Several alternative options including combined pathway inhibition to target MPNs are being explored.

\section{References}

1. Springuel L, Renauld JC, Knoops L (2015) JAK kinase targeting in hematologic malignancies: a sinuous pathway from identification of genetic alterations towards clinical indications. Haematologica 100(10): 1240-1253.

2. Hsu L, Armstrong AW (2014) JAK inhibitors: treatment efficacy and safety profile in patients with psoriasis. J Immunol Res 2014; 2014:1-7.

3. Sonbol MB, Firwana B, Zarzour A, Morad M, Rana V, et al. (2013) Comprehensive review of JAK inhibitors in myeloproliferative neoplasms. Ther Adv Hematol 4(1): 15-35.
4. Meyer SC, Levine RL (2014) Molecular pathways: molecular basis for sensitivity and resistance to JAK kinase inhibitors. Clin Cancer Res 20(8): 2051-2059.

5. Cervantes F, Pereira A (2017) Does ruxolitinib prolong the survival of patients with myelofibrosis? Blood 129(7): 832-837.

6. Spoerl S, Mathew NR, Bscheider M, Schmitt-Graeff A, Chen S, et al. (2014) Activity of therapeutic JAK $1 / 2$ blockade in graft-versus-host disease. Blood 123(24): 3832-3842.

7. Vannucchi AM, Guglielmelli P (2017) Traffic lights for ruxolitinib. Blood 130(9): 1075-1077.

8. Bose P, Verstovsek S (2016) Drug development pipeline for myeloproliferative neoplasms: potential future impact on guidelines and management. J Natl Compr Netw 14(12): 1613-1624.

9. Verstovsek S, Passamonti F, Rambaldi A, Barosi G, Rumi E, et al. (2017) Ruxolitinib for essential thrombocythemia refractory to or intolerant of hydroxyurea: long-term phase 2 study results. Blood 130(15): 17681771.

10. Finazzi G (2017) Ruxolitinib in ET: not all MPN are equal. Blood 130(17): $1873-1874$.

11. Lee S, Palmares C, Koutsoukos N, Weinberg B, Palmares T, et al. (2016) Ruxolitinib used to treat hypereosinophilic syndrome. Blood 128: 5500 .

\section{Your next submission with Juniper Publishers will reach you the below assets}

DOI: 10.19080/JTMP.2018.02.555598 